\title{
HOT WATER IN THE INNER 100 AU OF THE CLASS 0 PROTOSTAR NGC 1333 IRAS2A*
}

\author{
Ruud Visser ${ }^{1}$, Jes K. Jørgensen ${ }^{2,3}$, LARs E. Kristensen ${ }^{4,5}$, Ewine F. van Dishoeck ${ }^{4,6}$, And Edwin A. Bergin ${ }^{1}$ \\ ${ }^{1}$ Department of Astronomy, University of Michigan, 500 Church Street, Ann Arbor, MI 48109-1042, USA; visserr@ umich.edu \\ ${ }^{2}$ Niels Bohr Institute, University of Copenhagen, Juliane Maries Vej 30, DK-2100 Copenhagen Ø, Denmark \\ ${ }^{3}$ Centre for Star and Planet Formation, Natural History Museum of Denmark, University of Copenhagen, \\ Øster Voldgade 5-7, DK-1350 Copenhagen K, Denmark \\ ${ }^{4}$ Leiden Observatory, Leiden University, P.O. Box 9513, 2300 RA Leiden, The Netherlands \\ ${ }^{5}$ Harvard-Smithsonian Center for Astrophysics, 60 Garden Street, Cambridge, MA 02138, USA \\ ${ }^{6}$ Max-Planck-Institut für Extraterrestrische Physik, Giessenbachstrasse 1, D-85748 Garching, Germany \\ Received 2013 January 22; accepted 2013 March 25; published 2013 May 1
}

\begin{abstract}
Evaporation of water ice above $100 \mathrm{~K}$ in the inner few $100 \mathrm{AU}$ of low-mass embedded protostars (the so-called hot core) should produce quiescent water vapor abundances of $\sim 10^{-4}$ relative to $\mathrm{H}_{2}$. Observational evidence so far points at abundances of only a few $10^{-6}$. However, these values are based on spherical models, which are known from interferometric studies to be inaccurate on the relevant spatial scales. Are hot cores really that much drier than expected, or are the low abundances an artifact of the inaccurate physical models? We present deep velocity-resolved Herschel-HIFI spectra of the $3_{12}-3_{03}$ lines of $\mathrm{H}_{2}^{16} \mathrm{O}$ and $\mathrm{H}_{2}^{18} \mathrm{O}\left(1097 \mathrm{GHz}, E_{\mathrm{u}} / k=249 \mathrm{~K}\right)$ in the low-mass Class 0 protostar NGC 1333 IRAS2A. A spherical radiative transfer model with a power-law density profile is unable to reproduce both the HIFI data and existing interferometric data on the $\mathrm{H}_{2}^{18} \mathrm{O}_{13}-2_{20}$ line $\left(203 \mathrm{GHz}, E_{\mathrm{u}} / k=204 \mathrm{~K}\right)$. Instead, the HIFI spectra likely show optically thick emission from a hot core with a radius of about $100 \mathrm{AU}$. The mass of the hot core is estimated from the $\mathrm{C}^{18} \mathrm{O} J=9-8$ and 10-9 lines. We derive a lower limit to the hot water abundance of $2 \times 10^{-5}$, consistent with the theoretical predictions of $\sim 10^{-4}$. The revised $\mathrm{HDO} / \mathrm{H}_{2} \mathrm{O}$ abundance ratio is $1 \times 10^{-3}$, an order of magnitude lower than previously estimated.
\end{abstract}

Key words: astrochemistry - circumstellar matter - stars: formation - stars: protostars - techniques: spectroscopic

Online-only material: color figures

\section{INTRODUCTION}

In the embedded stage of low-mass star formation, the central source is surrounded by a collapsing envelope that spans a wide range in densities and temperatures (André et al. 2000). The inner region, known as the hot core or hot corino ( $T$ > 100 K; Walmsley \& Schilke 1992; van Dishoeck \& Blake 1998; Ceccarelli 2004), forms a crucial step in the flow of matter from the cold outer envelope toward the circumstellar disk (Visser et al. 2009). Thermal evaporation of water ice at about $100 \mathrm{~K}$ should produce a hot core water abundance of about $10^{-4}$ (Ceccarelli et al. 1996; Rodgers \& Charnley 2003). Such abundances are indeed observed for high-mass protostars (van der Tak et al. 2006; Chavarría et al. 2010; Herpin et al. 2012), though not universally (Emprechtinger et al. 2013). Abundances reported for low-mass hot cores have so far not exceeded $10^{-5}$ (Ceccarelli et al. 2000; Maret et al. 2002; Kristensen et al. 2010; Coutens et al. 2012). Are low-mass hot cores that much "drier" than expected?

Measuring the hot core water abundance in a protostar is not a trivial task, regardless of whether it is a low- or a highmass source. Shocked gas tends to outshine the quiescent inner envelope (Melnick et al. 2000; Chavarría et al. 2010; Kristensen et al. 2010, 2012; Coutens et al. 2012; Emprechtinger et al. 2013), so one has to isolate the envelope emission from velocityresolved spectra. Hot core abundances derived from spectrally unresolved data, such as from the Infrared Space Observatory (Ceccarelli et al. 2000; Maret et al. 2002), should therefore be treated with caution.

\footnotetext{
* Herschel is an ESA space observatory with science instruments provided by European-led Principal Investigator consortia and with important participation from NASA.
}

Another difficulty is the poorly known source structure on the spatial scales of the hot core: even if the column of quiescent hot water can be measured, there is no reliable column of hot $\mathrm{H}_{2}$ to compare against. Abundances of water and other molecules are typically computed by way of spherical envelope models with simple density profiles, constrained from single-dish dust continuum observations (Ceccarelli et al. 2000; van der Tak et al. 2000; Schöier et al. 2002; Maret et al. 2002; Coutens et al. 2012). Based on such a model, we previously derived a hot core abundance of $\leqslant 10^{-5}$ for the Class 0 protostar NGC 1333 IRAS2A (Kristensen et al. 2010; Liu et al. 2011). However, spherical models do not recover the density enhancements measured with continuum interferometry in the inner few $100 \mathrm{AU}$ of various low-mass protostars (Jørgensen et al. 2004, 2005, 2007; Chiang et al. 2008). The low water abundances may thus merely be an artifact of the inaccuracy of the adopted source models on the spatial scales of the hot core.

Using interferometric observations of the $3_{13}-2_{20}$ line of $\mathrm{H}_{2}^{18} \mathrm{O}$ at $203 \mathrm{GHz}\left(E_{\mathrm{u}} / k=204 \mathrm{~K}\right)$, Jørgensen \& van Dishoeck (2010b) and Persson et al. (2012) measured hot water column densities for the three Class 0 protostars NGC 1333 IRAS2A, $4 \mathrm{~A}$, and 4B. They also estimated $\mathrm{H}_{2}$ column densities from continuum interferometry on the same spatial scales to derive water abundances between a few $10^{-9}$ and a few $10^{-6}$. However, these abundances are relative to the total amount of gas in the inner $\sim 100 \mathrm{AU}$, not relative to just the gas above $100 \mathrm{~K}$. If a significant fraction of the material on these scales resides in an embedded disk or pseudo-disk, the bulk would be at lower temperatures and most water would be frozen out (Visser et al. 2009; Ilee et al. 2011).

In order to get additional constraints on the hot water in NGC 1333 IRAS2A, we performed a $5.1 \mathrm{hr}$ integration of the 
Table 1

Integrated Line Intensities ( $\int T_{\mathrm{mb}} d v$ in $\left.\mathrm{K} \mathrm{km} \mathrm{s}^{-1}\right)$ and $3 \sigma$ Upper Limits for Narrow Emission in NGC 1333 IRAS2A ${ }^{\mathrm{a}}$

\begin{tabular}{|c|c|c|c|c|c|c|c|c|c|}
\hline Species & Transition & $\begin{array}{l}\text { Telescope/ } \\
\text { Instrument }\end{array}$ & $\begin{array}{c}v \\
(\mathrm{GHz})\end{array}$ & $\begin{array}{c}E_{\mathrm{u}} / k \\
(\mathrm{~K})\end{array}$ & $\begin{array}{c}A_{\mathrm{ul}} \\
\left(\mathrm{s}^{-1}\right)\end{array}$ & $\begin{array}{c}\theta \\
\left({ }^{\prime \prime}\right)\end{array}$ & $\begin{array}{l}\text { Observed } \\
\text { Intensity }\end{array}$ & $\begin{array}{c}\text { Model } \\
\text { Intensity }\end{array}$ & Reference \\
\hline $\mathrm{p}-\mathrm{H}_{2}^{16} \mathrm{O}$ & $2_{02}-1_{11}$ & HIFI & 987.927 & 100.8 & $5.835(-3)$ & 21.5 & $<0.309$ & 0.312 & 1 \\
\hline $\mathrm{p}-\mathrm{H}_{2}^{16} \mathrm{O}$ & $2_{11}-2_{02}$ & HIFI & 752.033 & 136.9 & $7.062(-3)$ & 28.2 & $<0.583$ & 0.230 & 1 \\
\hline $\mathrm{o}-\mathrm{H}_{2}^{16} \mathrm{O}$ & $3_{12}-2_{21}$ & HIFI & 1153.127 & 249.4 & $2.634(-3)$ & 18.4 & $<0.545$ & 0.352 & 1 \\
\hline $\mathrm{o}-\mathrm{H}_{2}^{16} \mathrm{O}$ & $3_{12}-3_{03}$ & HIFI & 1097.365 & 249.4 & $1.648(-2)$ & 19.3 & $0.342 \pm 0.050^{\mathrm{b}}$ & 0.342 & 2 \\
\hline $\mathrm{p}-\mathrm{H}_{2}^{16} \mathrm{O}$ & $3_{31}-4_{04}$ & HIFI & 1893.687 & 410.4 & $1.630(-4)$ & 11.2 & $<0.531$ & 0.005 & 3 \\
\hline $\mathrm{p}-\mathrm{H}_{2}^{18} \mathrm{O}$ & $2_{02}-1_{11}$ & HIFI & 994.675 & 100.6 & $6.020(-3)$ & 21.3 & $<0.068$ & 0.005 & 1 \\
\hline $\mathrm{p}-\mathrm{H}_{2}^{18} \mathrm{O}$ & $3_{13}-2_{20}$ & PdBI & 203.408 & 203.7 & $4.812(-6)$ & 0.83 & $42 \pm 8$ & 42 & 4 \\
\hline $\mathrm{o}-\mathrm{H}_{2}^{18} \mathrm{O}$ & $3_{12}-3_{03}$ & HIFI & 1095.627 & 248.7 & $1.621(-2)$ & 19.4 & $0.078 \pm 0.011$ & 0.078 & 2 \\
\hline $\mathrm{o}-\mathrm{H}_{2}^{18} \mathrm{O}$ & $4_{14}-3_{21}$ & APEX & 390.608 & 322.0 & $3.143(-5)$ & 16.0 & $<0.410$ & $0.205^{\mathrm{c}}$ & 5 \\
\hline $\mathrm{o}-\mathrm{H}_{2}^{18} \mathrm{O}$ & $4_{23}-3_{30}$ & $\cdots$ & 489.054 & 429.6 & $6.887(-5)$ & 0.83 & $\ldots$ & 331 & $\cdots$ \\
\hline $\mathrm{o}-\mathrm{H}_{2}^{18} \mathrm{O}$ & $5_{32}-4_{41}$ & $\ldots$ & 692.079 & 727.6 & $1.478(-4)$ & 0.83 & $\ldots$ & 1.74 & $\ldots$ \\
\hline $\mathrm{p}-\mathrm{H}_{2}^{17} \mathrm{O}$ & $1_{11}-0_{00}$ & HIFI & 1107.167 & 53.1 & $1.812(-2)$ & 19.2 & $<0.037$ & 0.007 & 2 \\
\hline $\mathrm{o}-\mathrm{H}_{2}^{17} \mathrm{O}$ & $3_{12}-3_{03}$ & HIFI & 1096.414 & 249.1 & $1.635(-2)$ & 19.3 & $<0.043$ & 0.005 & 2 \\
\hline $\mathrm{C}^{18} \mathrm{O}$ & $9-8$ & HIFI & 987.560 & 237.0 & $7.380(-5)$ & 21.5 & $0.157 \pm 0.032$ & $0.043^{\mathrm{d}}$ & 6 \\
\hline $\mathrm{C}^{18} \mathrm{O}$ & $10-9$ & HIFI & 1097.163 & 289.7 & $9.661(-5)$ & 19.3 & $0.150 \pm 0.050$ & $0.035^{\mathrm{d}}$ & 2 \\
\hline
\end{tabular}

Notes.

a Also listed are the frequency $(\nu)$, upper-level energy $\left(E_{\mathrm{u}}\right)$, Einstein $A$ coefficient $\left(A_{\mathrm{ul}}\right)$, and beam diameter $(\theta)$ for each line. The notation $a(-b)$ denotes $a \times 10^{-b}$. The model intensities are corrected for beam dilution and dust extinction as described in Section 4.3.

b $\int T_{\mathrm{mb}} d v$ for the broad and medium components is $3.34 \pm 0.09$ and $1.44 \pm 0.08 \mathrm{~K} \mathrm{~km} \mathrm{~s}^{-1}$.

c The intensity predicted for a fictitious $0^{\prime \prime} 83$ ALMA beam is $76.4 \mathrm{~K} \mathrm{~km} \mathrm{~s}^{-1}$.

${ }^{\mathrm{d}}$ For an $\mathrm{H}_{2} \mathrm{O} / \mathrm{CO}$ ratio of unity above $100 \mathrm{~K}$. Most of the observed $\mathrm{C}^{18} \mathrm{O}$ emission originates in gas below $100 \mathrm{~K}$, which is not included in the model.

References. (1) Kristensen et al. 2010; (2) this work; (3) A. O. Benz et al. (in preparation); (4) Persson et al. 2012; (5) F. Wyrowski (2012, private communication); (6) Y1ldız et al. 2010.

$3_{12}-3_{03}$ lines of $\mathrm{H}_{2}^{16} \mathrm{O}, \mathrm{H}_{2}^{17} \mathrm{O}$, and $\mathrm{H}_{2}^{18} \mathrm{O}$ at $1097 \mathrm{GHz}$ with the Heterodyne Instrument for the Far-Infrared (HIFI; de Graauw et al. 2010) on the Herschel Space Observatory (Pilbratt et al. 2010). This observation is part of the key program "Water in star-forming regions with Herschel" (WISH; van Dishoeck et al. 2011), which aims to study the physics and chemistry of water during star formation across a range of masses and evolutionary stages. HIFI has high enough spectral resolution to disentangle the quiescent and shocked gas, and the deep integration allows for a detection of $\mathrm{H}_{2}^{18} \mathrm{O}$.

This paper presents the observations (Sections 2 and 3 ) along with a detailed analysis (Section 4). We develop a scenario in which the observed emission originates in the same gas as the $203 \mathrm{GHz}$ line, namely in a hot core with a radius of about 100 AU. Our definition of a "hot core" is fairly loose and includes all quiescent (i.e., non-shocked) material hotter than $100 \mathrm{~K}$, regardless of whether it is part of the envelope or an embedded disk-like structure. A simple spherical model fails at reproducing both data sets simultaneously, so we perform a model-independent analysis to constrain the distribution of water within the hot core. Comparing the water column density against Herschel-HIFI $\mathrm{C}^{18} \mathrm{O} J=9-8$ and $10-9$ data yields a lower limit on the water abundance of $2 \times 10^{-5}$ relative to $\mathrm{H}_{2}$. As concluded in Section 5, the hot core in IRAS2A does not appear to be as dry as previously estimated from pure spherical models.

\section{OBSERVATIONS AND DATA REDUCTION}

NGC 1333 IRAS2A is a low-mass Class 0 protostar $\left(L_{\mathrm{bol}}=\right.$ $35.7 L_{\odot}, T_{\text {bol }}=50 \mathrm{~K}$; Kristensen et al. 2012) located at a distance of $235 \mathrm{pc}$ in Perseus at coordinates $3^{\mathrm{h}} 28^{\mathrm{m}} 55^{\mathrm{s}} .6$ by $+31^{\circ} 14^{\prime} 37^{\prime \prime}$. 1 (J2000; Hirota et al. 2008). Based on large-scale CO 3-2 maps, it is oriented close to edge on (Sandell et al. 1994).
IRAS2A was observed on 2011 March 12 (ObsID 1342215968) with HIFI on Herschel (Pilbratt et al. 2010; de Graauw et al. $2010)$ in double beam switch mode with a nod of $3^{\prime}$. The primary targets were the $3{ }_{12}-3_{03}$ lines of ortho- $\mathrm{H}_{2}^{16} \mathrm{O}$ and $\mathrm{H}_{2}^{18} \mathrm{O}$ at 1097.365 and $1095.627 \mathrm{GHz}$ in band $4\left(E_{\mathrm{u}} / k=249 \mathrm{~K}\right)$. The full spectrum covers a range of $4 \mathrm{GHz}$ in each sideband, including the $3_{12}-3_{03}$ and $1_{11}-0_{00}$ lines of $\mathrm{H}_{2}^{17} \mathrm{O}$ and the $10-9$ line of $\mathrm{C}^{18} \mathrm{O}$ (Table 1). The data were recorded in $\mathrm{H}$ and $\mathrm{V}$ polarization using both the wide-band spectrometer (WBS; $1.1 \mathrm{MHz}$ resolution) and the high-resolution spectrometer (HRS; $0.5 \mathrm{MHz}$ ). The HIFI beam size at $1100 \mathrm{GHz}$ is $19^{\prime \prime} .3$ and the overall flux uncertainty is about $10 \%$ (Roelfsema et al. 2012).

The $3_{12}-3_{03}$ line of $\mathrm{H}_{2}^{16} \mathrm{O}$ was previously detected in IRAS2A after a 30 minute integration (Kristensen et al. 2010). The current observation had a total exposure time of $5.1 \mathrm{hr}$ in order to detect the $\mathrm{H}_{2}^{18} \mathrm{O}$ line and to improve the decomposition of the various velocity components in the $\mathrm{H}_{2}^{16} \mathrm{O}$ spectrum (Section 3). The longer integration time also puts a more sensitive upper limit on the $\mathrm{H}_{2}^{17} \mathrm{O}$ line.

The data were reduced with HIPE v8.2.1 (Ott 2010) and exported to CLASS ${ }^{7}$ for analysis. The intensities were converted from antenna to main-beam temperature scale through a mainbeam efficiency of 0.74 (Roelfsema et al. 2012). The $H$ and $V$ spectra were averaged after individual inspection. The final data reduction step was to subtract a linear baseline.

\section{RESULTS}

\subsection{New Data}

The full HIFI spectrum as recorded with the WBS back end is shown in Figures 6 and 7 in the Appendix. The strongest feature

\footnotetext{
7 http://www.iram.fr/IRAMFR/GILDAS
} 


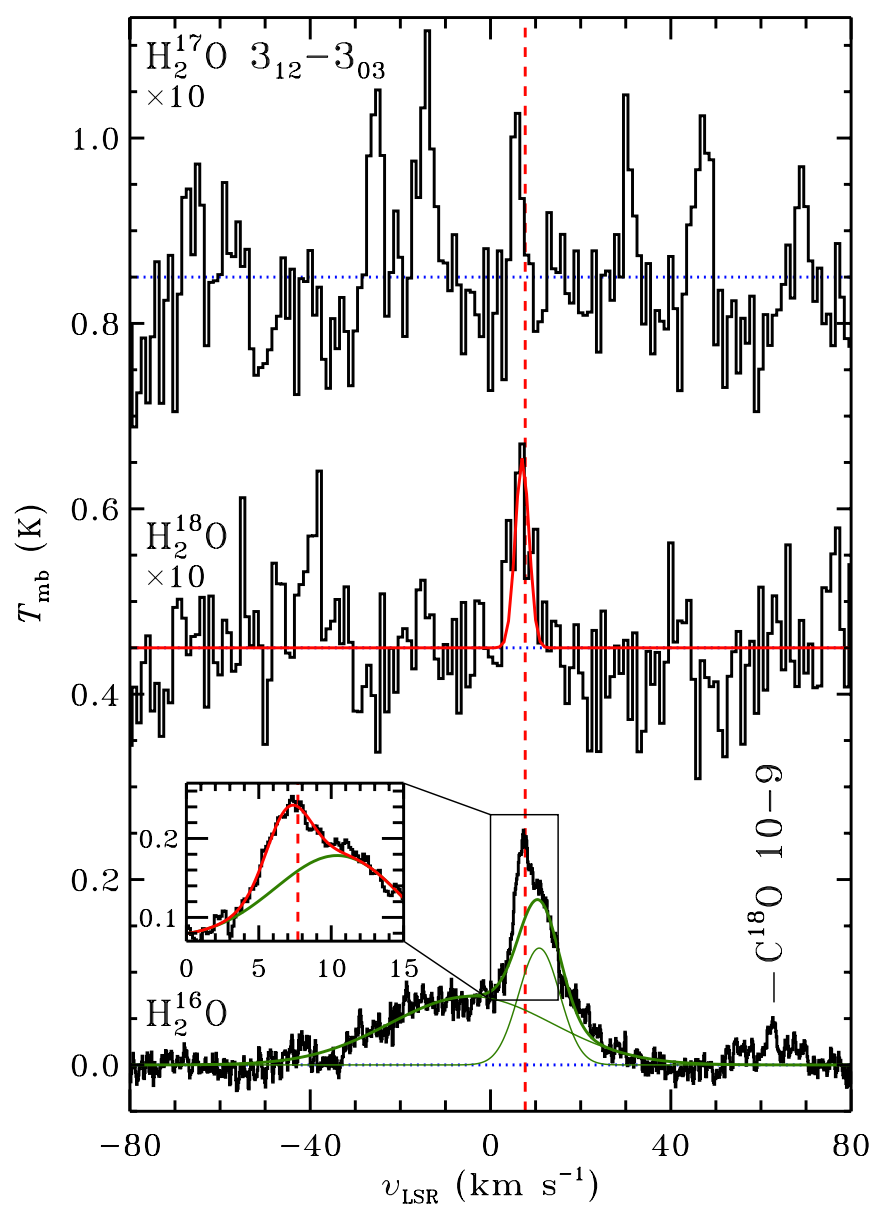

Figure 1. Baseline-subtracted spectra of the $3_{12}-3_{03}$ lines of $\mathrm{H}_{2}^{16} \mathrm{O}$ (bottom), $\mathrm{H}_{2}^{18} \mathrm{O}$ (middle), and $\mathrm{H}_{2}^{17} \mathrm{O}$ (top) in NGC 1333 IRAS2A. The $\mathrm{H}_{2}^{16} \mathrm{O}$ spectrum includes Gaussian profiles (green) fitted to the broad and medium components. Shown as an inset is a blow-up of the narrow feature in the $\mathrm{H}_{2}^{16} \mathrm{O}$ spectrum, with a Gaussian fitted in red. The narrow $\mathrm{H}_{2}^{18} \mathrm{O}$ feature is also fitted with a red Gaussian. The vertical dashed red lines mark the source velocity of $7.7 \mathrm{~km} \mathrm{~s}^{-1}$. The $\mathrm{H}_{2}^{16} \mathrm{O}$ spectrum is shown at the original spectral resolution of $0.14 \mathrm{~km} \mathrm{~s}^{-1}$. The other two spectra are rebinned to $1.0 \mathrm{~km} \mathrm{~s}^{-1}$ and blown up by a factor of 10 .

(A color version of this figure is available in the online journal.)

is the $3_{12}-3_{03}$ line of $\mathrm{H}_{2}^{16} \mathrm{O}$, peaking at $230 \mathrm{mK}$. The $3_{12}-3_{03}$ spectrum for each water isotopolog is plotted in Figure 1. Thanks to the excellent signal-to-noise ratio from the long integration ( $8 \mathrm{mK} \mathrm{rms}$ in $0.5 \mathrm{~km} \mathrm{~s}^{-1}$ bins), the $\mathrm{H}_{2}^{16} \mathrm{O}$ spectrum now shows a distinct narrow emission feature on top of the medium and broad features detected by Kristensen et al. (2010). The three components have full widths at half-maximum (FWHMs) of $42.5 \pm 0.8,10.7 \pm 0.4$, and $3.6 \pm 0.3 \mathrm{~km} \mathrm{~s}^{-1}$. Table 1 lists the integrated intensities. The broad and medium FWHMs are the same as those reported by Kristensen et al. for lower-excitation water lines. These components arise in shocked gas and are not considered here any further.

The $\mathrm{H}_{2}^{18} \mathrm{O}$ spectrum shows a weak emission feature $(7 \sigma)$ in both polarization filters, with the same intensity in the WBS and HRS recordings. The JPL and CDMS spectral line databases (Pickett et al. 1998; Müller et al. 2001) show no other plausible features at this position in either the upper or the lower sideband. Figure 1 shows a Gaussian fit with an FWHM fixed at $3.6 \mathrm{~km} \mathrm{~s}^{-1}$ (taken from the $\mathrm{H}_{2}^{16} \mathrm{O}$ decomposition), because the feature is too weak for a reliable independent measurement of its width.
The constrained fit has a peak intensity of $20 \pm 4 \mathrm{mK}$ and an integrated intensity of $78 \pm 11 \mathrm{mK} \mathrm{km} \mathrm{s}^{-1}$ (Table 1).

The $\mathrm{H}_{2}^{17} \mathrm{O}$ spectrum in Figure 1 shows an apparent emission feature blueshifted by $2 \mathrm{~km} \mathrm{~s}^{-1}$ from the source velocity, along with four features of similar strength at larger offsets. Three of them could represent lines of $\mathrm{CH}_{2} \mathrm{DOH}\left(E_{\mathrm{u}} / k=\right.$ 229-294 K), which has been detected before in single-dish spectra of IRAS2A (Parise et al. 2006; see also Figures 6 and 7). The JPL and CDMS databases offer no plausible identification for the fourth feature. We consider the $3_{12}-3_{03}$ line undetected in $\mathrm{H}_{2}^{17} \mathrm{O}$, but our analysis in Section 4 is unaffected by the question of whether it is real or not. The potential $\mathrm{CH}_{2} \mathrm{DOH}$ lines are not considered any further.

The only other confirmed feature in the entire spectral setting is the $\mathrm{C}^{18} \mathrm{O} 10-9$ line at $1097.163 \mathrm{GHz}$. It appears to be a blend of a narrow and a medium component, but the detection is too weak for an accurate decomposition. The intensity integrated over the full profile is $300 \pm 30 \mathrm{mK} \mathrm{km} \mathrm{s}^{-1}$, split roughly halfway between the two components. Narrow and medium features have been detected previously in various low- and high$J$ CO isotopolog lines (Yildiz et al. 2010). The $\mathrm{C}^{18} \mathrm{O} 10-9$ line is analyzed as part of a larger sample of lines and sources by San Jose-Garcia et al. (2013) and Yildiz et al. (2013).

The full spectrum in Figures 6 and 7 appears to contain a few more lines, but their nature is uncertain and not of interest to this paper. We merely mention two possible identifications: $\mathrm{H}_{2} \mathrm{CO}$ at $1094.590 \mathrm{GHz}\left(E_{\mathrm{u}} / k=526 \mathrm{~K}\right)$ and $\mathrm{CH}_{3} \mathrm{OH}$ at $1095.063 \mathrm{GHz}$ $\left(E_{\mathrm{u}} / k=498 \mathrm{~K}\right)$. Both species have been detected previously in single-dish and interferometric studies of IRAS2A (Maret et al. 2005; Parise et al. 2006; Jørgensen et al. 2007).

\subsection{Complementary Data}

IRAS2A has been targeted in various other water lines with Herschel. Visser et al. (2012) detected about a dozen spectrally unresolved lines with the PACS instrument (60-180 micron; $E_{\mathrm{u}} / k$ up to $1750 \mathrm{~K}$ ), attributed to shocked gas on 100-1000 AU scales and unrelated to the narrow emission analyzed here. No narrow emission has been detected in any of the water lines previously observed with HIFI (Kristensen et al. 2010). Upper limits on such emission may be useful and are reported in Table 1. Another HIFI upper limit is available for $\mathrm{H}_{2}^{16} \mathrm{O} 3_{31}-4_{04}$ at $1894 \mathrm{GHz}$, observed alongside $\mathrm{C}^{+}{ }^{2} \mathrm{P}_{3 / 2}-{ }^{2} \mathrm{P}_{1 / 2}$ (A. O. Benz et al., in preparation). In most cases, the upper limit in Table 1 is the $3 \sigma$ limit computed from the rms noise in $0.5 \mathrm{~km} \mathrm{~s}^{-1}$ bins. This method does not work for the $\mathrm{H}_{2}^{16} \mathrm{O} 2_{02}-1_{11}$ and $2_{11}-2_{02}$ lines, where the broad and medium emission features can hide a narrow feature stronger than three times the noise. Instead, we forced a three-Gaussian fit on these spectra, with the position and width of the narrow feature fixed at the values found for the $3_{12}-3_{03}$ line. The adopted upper limit is twice the integrated intensity of the force-fitted narrow component. In doing so, we assume the broad and medium components do not shield any narrow emission. The $1_{10}-1_{01}, 1_{11}-0_{00}$, and $2_{12}-1_{01}$ lines show narrow absorption due to cold water vapor in the outer envelope (Kristensen et al. 2010) and are excluded from our analysis.

Ground-based observations exist for two $\mathrm{H}_{2}^{18} \mathrm{O}$ lines: $3_{13}-2_{20}$ at $203 \mathrm{GHz}\left(E_{\mathrm{u}} / k=204 \mathrm{~K}\right)$ and $4_{14}-3_{21}$ at $391 \mathrm{GHz}$ $\left(E_{\mathrm{u}} / k=322 \mathrm{~K}\right)$. The latter was undetected with APEX in a $16^{\prime \prime}$ beam, with a $3 \sigma$ upper limit of $410 \mathrm{mK} \mathrm{km} \mathrm{s}^{-1}$ (Table 1; F. Wyrowski 2012, private communication). Persson et al. (2012) detected spatially resolved compact and extended emission in the $3_{13}-2_{20}$ line with the Plateau de Bure Interferometer $(\mathrm{PdBI})$. The extended component is associated with the outflow 
and is ignored here. The compact component has an FWHM of $4.0 \pm 0.1 \mathrm{~km} \mathrm{~s}^{-1}$, comparable to the narrow component in the $\mathrm{H}_{2}^{16} \mathrm{O} 3_{12}-3_{03}$ spectrum in Figure 1. The compact PdBI component has an integrated intensity of $0.98 \mathrm{Jy} \mathrm{km} \mathrm{s}{ }^{-1}$ or $42 \mathrm{~K} \mathrm{~km} \mathrm{~s}^{-1}$. Its spatial extent was marginally resolved to a diameter of 0.83 , probing material out to a radius of $100 \mathrm{AU}$. Persson et al. attributed the compact emission to a flattened inner envelope or pseudo-disk dominated by infall rather than rotation.

\section{ANALYSIS}

\subsection{Spherical Model}

The HIFI $3{ }_{12}-3_{03}$ lines have upper-level energies of $249 \mathrm{~K}$. The bulk of the water in IRAS2A is present at temperatures below $100 \mathrm{~K}$, where it generally exists as ice rather than vapor. The low gas-phase abundance in the cold envelope $\left(\sim 10^{-8}\right.$; Kristensen et al. 2010; Liu et al. 2011), coupled with the low excitation temperatures, falls orders of magnitude shy of reproducing the observed narrow intensities (van Kempen et al. 2008).

That leaves two options for the narrow $3_{12}-3_{03}$ emission: a maser or the hot core. Furuya et al. (2003) observed maser emission toward IRAS2A at $22 \mathrm{GHz}$, with intensities varying by an order of magnitude on timescales of about a year. The maser lines were seen sometimes at the source velocity and sometimes blueshifted by a few km s${ }^{-1}$, with FWHMs from 1.2 to $2.3 \mathrm{~km} \mathrm{~s}^{-1}$. The HIFI $3_{12}-3_{03}$ and PdBI $3_{13}-2_{20}$ spectra show no such blueshifted emission and the emission at the source velocity is 2-3 times as broad as the $22 \mathrm{GHz}$ maser lines. Furthermore, our two epochs of HIFI data (Figure 1 and Kristensen et al. 2010) show no signs of variability on a one-year baseline. Hence, we conclude that the narrow $3_{12}-3_{03}$ emission is not associated with maser activity. Persson et al. (2012) employed similar arguments to reach the same conclusion for their PdBI data.

Instead, the narrow HIFI and PdBI lines are thermal and likely originate in the inner $100 \mathrm{AU}$ of IRAS2A, in quiescent gas above $100 \mathrm{~K}$. If the circumstellar material in IRAS2A is approximated as a spherical envelope with a power-law density profile, the $100 \mathrm{~K}$ radius lies at $94 \mathrm{AU}$ (Kristensen et al. 2012). While this matches the size of the emitting region seen with the PdBI, continuum interferometry of IRAS2A has shown that the assumptions of spherical symmetry and a single power-law density profile break down inside a radius of $1^{\prime \prime} .5$ or $350 \mathrm{AU}$ (Jørgensen et al. 2004, 2005).

A simple test reveals that the spherical model also fails to reproduce the HIFI and PdBI data simultaneously. The test consists of synthesizing the $3_{12}-3_{03}$ and $3_{13}-2_{20}$ spectra from a step abundance profile, as described in detail by van Kempen et al. (2008). Kristensen et al. (2012) constructed a source model for IRAS2A to fit the spectral energy distribution and submillimeter brightness profiles. The model envelope has a total mass of $5.1 M_{\odot}$, distributed along an $r^{-1.7}$ power law. The temperatures of the gas and dust are coupled and decrease from $250 \mathrm{~K}$ at the inner edge $(36 \mathrm{AU})$ to $10 \mathrm{~K}$ at the outer edge (17,000 AU). The step abundance profile consists of a high abundance $X_{\text {in }}$ above $100 \mathrm{~K}$ (at $94 \mathrm{AU}$ ) and a lower abundance $X_{\text {out }}$ below $100 \mathrm{~K}$. The HIFI and PdBI emission originate above $100 \mathrm{~K}$, so the question we want to answer is whether the spherical envelope model can reproduce both data sets with a single $X_{\text {in }}$.

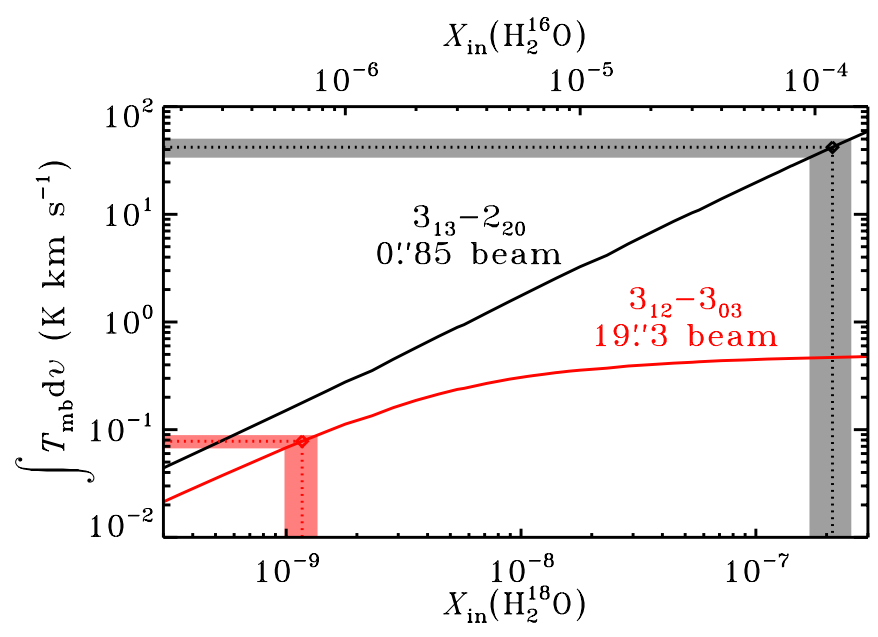

Figure 2. Integrated intensities for the HIFI $3_{12}-3_{03}$ line (red) and the PdBI $3_{13}-2_{20}$ line (black) of $\mathrm{H}_{2}^{18} \mathrm{O}$ in the spherical envelope model of Kristensen et al. (2012). The intensities, convolved to the appropriate beam size, are plotted as function of the hot core abundance of $\mathrm{H}_{2}^{18} \mathrm{O}$ (bottom axis) or $\mathrm{H}_{2}^{16} \mathrm{O}$ (top axis) for a water ortho/para ratio of 3 . The horizontal dotted lines and bars mark the observed intensities and $1 \sigma$ uncertainties. The abundances required to fit each observation individually are indicated by the vertical dotted lines and bars.

(A color version of this figure is available in the online journal.)

The molecular excitation and line emission are computed with the one-dimensional radiative transfer code RATRAN (Hogerheijde \& van der Tak 2000). This is a standard approach for interpreting molecular line spectra; see, e.g., Yildız et al. (2010), Liu et al. (2011), and Coutens et al. (2012) for recent examples of $\mathrm{C}^{18} \mathrm{O}, \mathrm{HDO}$, and $\mathrm{H}_{2} \mathrm{O}$. We set the gas-to-dust ratio to 100 and use OH5 opacities (Ossenkopf \& Henning 1994), appropriate for dust grains with thin ice mantles. The ortho/ para ratio of $\mathrm{H}_{2}$ is thermalized and that of water is fixed at 3 . Collision rates are taken from Dubernet et al. $(2006,2009)$ and Daniel et al. $(2010,2011)$ as compiled in the LAMDA database (Schöier et al. 2005). The outer $\mathrm{H}_{2}^{18} \mathrm{O}$ abundance does not affect the $3_{12}-3_{03}$ and $3_{13}-2_{20}$ lines and is fixed at $2 \times 10^{-11}$, derived from absorption seen with HIFI in lower rotational lines of water (Kristensen et al. 2010; Liu et al. 2011). The inner $\mathrm{H}_{2}^{18} \mathrm{O}$ abundance is varied from $3 \times 10^{-10}$ to $3 \times 10^{-7}\left(2 \times 10^{-7}\right.$ to $2 \times 10^{-4}$ for $\mathrm{H}_{2}^{16} \mathrm{O}$ ) to cover the full range from a "dry" to a "wet" hot core. Finally, the synthetic spectra are convolved to the appropriate beam size and compared to the available observations.

Figure 2 shows the convolved intensities for the $\mathrm{H}_{2}^{18} \mathrm{O} 3_{12}-3_{03}$ and $33_{13}-2_{20}$ lines as function of the inner abundance. The horizontal dotted lines and bars mark the observed intensities and $1 \sigma$ uncertainties: red for $3_{12}-3_{03}$ and black for $3_{13}-2_{20}$. The abundances required to fit each line individually are marked by the vertical dotted lines and bars. Within the confines of the spherical envelope model, the $\mathrm{H}_{2}^{18} \mathrm{O} 3_{12}-3_{03}$ spectrum from Figure 1 requires an inner $\mathrm{H}_{2}^{18} \mathrm{O}$ abundance of $1.2 \times 10^{-9}$. However, this underproduces the observed PdBI line intensity by a factor of 200. Matching the PdBI line requires a much higher inner $\mathrm{H}_{2}^{18} \mathrm{O}$ abundance of $2.1 \times 10^{-7}$, but then the $3_{12}-3_{03}$ line comes out a factor of six too strong. The estimated uncertainty on the $3_{12}-3_{03}$ intensity for $\mathrm{H}_{2}^{18} \mathrm{O}$ is $17 \%(10 \%$ calibration, $14 \%$ statistical; Sections 2 and 3.1), so a factor of six is $35 \sigma$ away. The RATRAN test clearly fails: a spherical envelope model with a single power-law density profile, such as used in previous attempts to derive hot core water abundances, 
cannot reproduce the single-dish and interferometric hot water observations simultaneously.

\subsection{LTE Analysis}

The mismatch of best-fit abundances to single-dish and interferometric data has also been seen for methanol in IRAS2A (Jørgensen et al. 2005) and for deuterated water in the Class 0 protostar IRAS 16293-2422 (Coutens et al. 2012; Persson et al. 2013). We thus have several pieces of evidence that simple spherical models break down on the spatial scales of the hot core. Until more appropriate source models can be generated-for example from sub-arcsecond PdBI and ALMA observations of protostellar dust and gas - it is better to take a step back and see what we can learn about hot water in IRAS2A irrespective of the underlying density and temperature structure.

\subsubsection{PdBI Emission: Optically Thin}

In analyzing the PdBI data, Persson et al. (2012) assumed optically thin emission in local thermodynamic equilibrium (LTE) and considered gas temperatures between 50 and $250 \mathrm{~K}$. The $3_{13}-2_{20}$ line has a critical density of a few $10^{5} \mathrm{~cm}^{-3}$. The critical density of the HIFI $3_{12}-3_{03}$ line is about a factor of 1000 higher, but with densities in the inner envelope easily exceeding $10^{9} \mathrm{~cm}^{-3}$ (Visser et al. 2009), the assumption of LTE is probably justified and we adopt it here as well.

The dust and gas temperatures are likely coupled at these high densities, so temperatures below $100 \mathrm{~K}$ can be excluded because of freeze-out. This also means that the 0.83 emitting region resolved with the $\mathrm{PdBI}$ represents the entire reservoir of quiescent (i.e., non-shocked) hot gas in IRAS2A. The PdBI observations have a field of view of $25^{\prime \prime}$, a little larger than the HIFI beam of $19^{\prime \prime}$ at $1097 \mathrm{GHz}$. Hence, if any other hot gas had been present within the HIFI beam but outside the central 0.'83, it would have been detected with the PdBI.

Following Goldsmith \& Langer (1999), the optical depth of a molecular line is

$$
\tau_{v}=\frac{N_{\mathrm{u}}}{\Delta v} \frac{A_{\mathrm{ul}} c^{3}}{8 \pi v^{3}}\left(e^{h v / k T}-1\right),
$$

with $v$ the frequency of the transition, $A_{\mathrm{ul}}$ the Einstein $A$ coefficient, and $\Delta v$ the observed FWHM. In LTE, the upperlevel column density $N_{\mathrm{u}}$ is

$$
N_{\mathrm{u}}=\frac{N g_{\mathrm{u}}}{Q(T)} e^{-E_{\mathrm{u}} / k T},
$$

with $N$ the total column density, $g_{\text {u }}$ the degeneracy, and $Q$ the partition function. The PdBI line is optically thin with $\tau_{v} \approx 0.1$. For a water ortho/para ratio of 3 , the observed PdBI intensity requires $N\left(\mathrm{H}_{2}^{18} \mathrm{O}\right) \approx 3 \times 10^{16} \mathrm{~cm}^{-2}$ regardless of the exact temperature between 100 and $250 \mathrm{~K}$ (Persson et al. 2012). The $3_{12}-3_{03}$ lines have Einstein $A$ coefficients a few thousand times faster than the $3_{13}-2_{20}$ line (Table 1) and are optically thick for all three isotopologs. For example, at $100 \mathrm{~K}, \tau_{v}=9.7$ for $\mathrm{H}_{2}^{17} \mathrm{O}$, 34 for $\mathrm{H}_{2}^{18} \mathrm{O}$, and 19,000 for $\mathrm{H}_{2}^{16} \mathrm{O}$. The optical depths decrease by about $50 \%$ if the temperature is increased to $250 \mathrm{~K}$.

\subsubsection{HIFI Emission: Optically Thick}

If the HIFI emission in all three isotopologs is indeed optically thick and characterized by the same temperature, the $3_{12}-3_{03}$ lines would have had the same intensities. This is clearly inconsistent with the data (Figure 1). However, the size of the

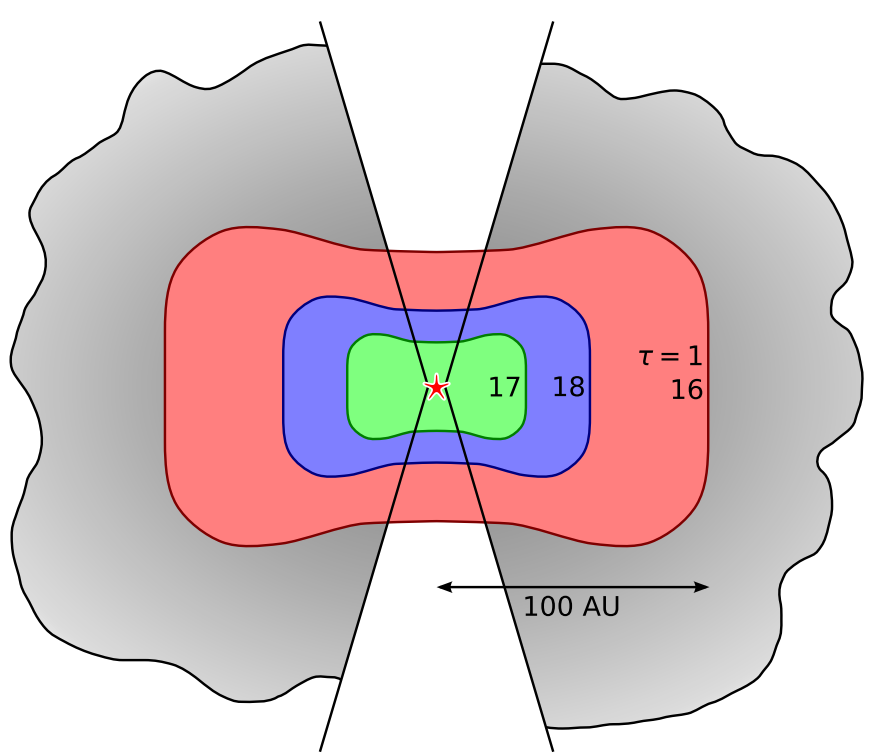

Figure 3. Illustration of the hot core $(T>100 \mathrm{~K})$ in IRAS2A. The red and blue areas show the $\tau_{v}=1$ surfaces for the $3_{12}-3_{03}$ lines of $\mathrm{H}_{2}^{16} \mathrm{O}$ and $\mathrm{H}_{2}^{18} \mathrm{O}$, with a difference in area that explains the different intensities observed with Herschel-HIFI (Figure 1). The green area shows the maximum size of the $\mathrm{H}_{2}^{17} \mathrm{O}$ $\tau_{v}=1$ surface allowed by the $3 \sigma$ upper limit from HIFI. The red area is the same as that of the spatially resolved $\mathrm{H}_{2}^{18} \mathrm{O} 3_{13}-2_{20}$ emission detected with the PdBI (Persson et al. 2012). The hot core is embedded inside a colder envelope ( $T<100 \mathrm{~K}$; shaded gray), extending out to $10^{4} \mathrm{AU}$ (not drawn to scale).

(A color version of this figure is available in the online journal.)

optically thick region (approximately the $\tau_{v}=1$ surface) may be different for each isotopolog, leading to different beam-filling factors. As argued below, this can explain the factor of 4.4 difference in observed intensities for $\mathrm{H}_{2}^{16} \mathrm{O}$ and $\mathrm{H}_{2}^{18} \mathrm{O}$ as well as the non-detection of $\mathrm{H}_{2}^{17} \mathrm{O}$.

Figure 3 shows a sketch of the flattened inner envelope of IRAS2A with the different emitting areas for the optically thick $3_{12}-3_{03}$ lines. The $\mathrm{H}_{2}^{18} \mathrm{O} 3_{13}-2_{20}$ line observed with the PdBI is optically thin and traces the entire hot core to its outer radius of $100 \mathrm{AU}$. The extreme optical depth of 19,000 for $\mathrm{H}_{2}^{16} \mathrm{O} 3_{12}-3_{03}$ means that its $\tau_{v}=1$ surface, measured from the outside, essentially lies right at the hot core's outer edge. $\mathrm{H}_{2}^{16} \mathrm{O}$ therefore emits at close to $100 \mathrm{~K}$. This is somewhat lower than the excitation temperature of $170 \mathrm{~K}$ assumed by Persson et al. (2012), but the difference has no substantial effect on our analysis. The temperature structure on these small scales is unknown, so we assume for simplicity that $\mathrm{H}_{2}^{18} \mathrm{O}$ and $\mathrm{H}_{2}^{17} \mathrm{O}$ also emit at $100 \mathrm{~K}$. The radius of the $\mathrm{H}_{2}^{18} \mathrm{O} \tau_{v}=1$ surface then has to be a factor of $\sqrt{4.4}=2.1$ smaller $(r \approx 47 \mathrm{AU})$ than that of $\mathrm{H}_{2}^{16} \mathrm{O}$ (Figure 3 ). The size difference would be smaller if we take a higher temperature for $\mathrm{H}_{2}^{18} \mathrm{O}$, but the available data do not allow for a more precise estimate.

\subsubsection{Water Distribution}

For an $\mathrm{H}_{2}^{18} \mathrm{O}$ column density of $3 \times 10^{16} \mathrm{~cm}^{-2}$, the optical depth in the $\mathrm{H}_{2}^{18} \mathrm{O} 3_{12}-3_{03}$ line is 34 at $100 \mathrm{~K}$ (Equation (1)). If the water is distributed uniformly throughout the hot core, the $\tau_{v}=1$ surface for $\mathrm{H}_{2}^{18} \mathrm{O}$ would lie at $1 / 34$ th of the way in from the outer edge at $100 \mathrm{AU}$, i.e., at $r \approx 97 \mathrm{AU}$. The surface has to be at $r \approx 47 \mathrm{AU}$ to explain the observed $\mathrm{H}_{2}^{16} \mathrm{O} / \mathrm{H}_{2}^{18} \mathrm{O}$ intensity ratio from Figure 1, so the water cannot be distributed uniformly with radius. 


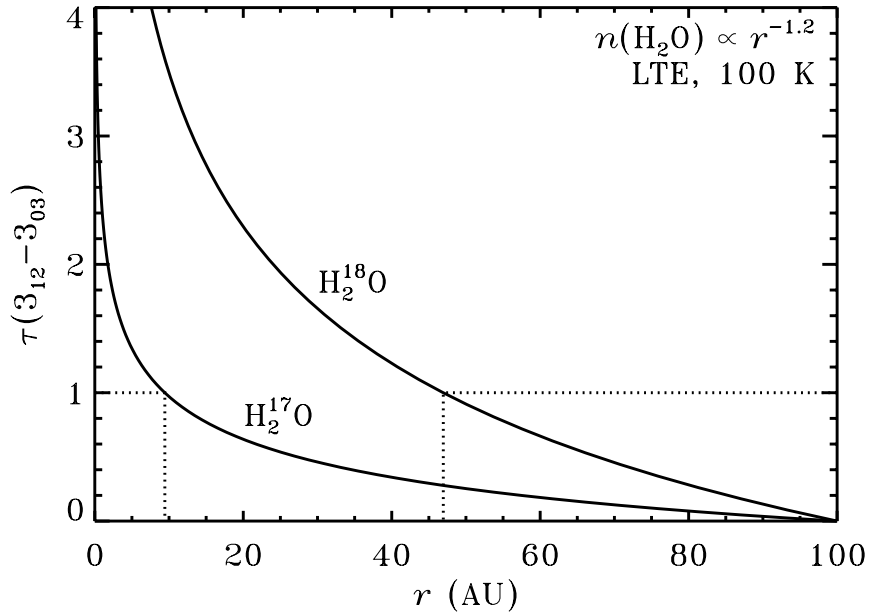

Figure 4. Optical depth of $\mathrm{H}_{2}^{18} \mathrm{O}$ and $\mathrm{H}_{2}^{17} \mathrm{O} 3_{12}-3_{03}$ measured inward from the outer edge of the hot core (Figure 3 ). Both curves are for power-law water density profiles at an assumed emitting temperature of $100 \mathrm{~K}$. The dotted lines mark the $\tau_{v}=1$ radii.

A power-law distribution, $n\left(\mathrm{H}_{2} \mathrm{O}\right) \propto r^{-p}$, offers a simple solution. This does not necessarily imply an abundance gradient, because it can be the total gas density itself that increases toward smaller radii (Visser et al. 2009). We need to know the inner radius of the water reservoir in order to compute the power-law exponent $p$, but the inner radius cannot be measured from the $\mathrm{PdBI}$ data. A rough estimate would be $0.1 \mathrm{AU}$, the typical inner edge of accretion disks around T Tauri stars (Akeson et al. 2005). The requirement that only $1 / 34$ th $(3 \%)$ of the $\mathrm{H}_{2}^{18} \mathrm{O}$ column lie at $r>47 \mathrm{AU}$ then yields $p=1.2$. The slope steepens to 1.4 if the inner radius is placed at $1 \mathrm{AU}$ instead.

Figure 4 shows the resulting optical depth profiles ( $\tau_{v}$ as function of $r$ ) for $\mathrm{H}_{2}^{18} \mathrm{O}$ and $\mathrm{H}_{2}^{17} \mathrm{O}$. The $\tau_{v}=1$ surface for $\mathrm{H}_{2}^{16} \mathrm{O}$ (not plotted) lies at $100 \mathrm{AU}$, consistent with Figure 3. $\mathrm{H}_{2}^{18} \mathrm{O}$ reaches $\tau_{v}=1$ at $47 \mathrm{AU}$, as needed to fit the $3_{12}-3_{03}$ observations. The $\tau_{v}=1$ surface for the least abundant isotopolog, $\mathrm{H}_{2}^{17} \mathrm{O}$, ends up at $9.4 \mathrm{AU}$, providing 110 times more beam dilution than experienced by $\mathrm{H}_{2}^{16} \mathrm{O}$. This places the observable $\mathrm{H}_{2}^{17} \mathrm{O} 3_{12}-3_{03}$ intensity about a factor of 10 below the $3 \sigma$ upper limit from Figure 1 and Table 1.

\subsubsection{Summary of LTE Analysis}

In summary, the $3_{12}-3_{03}$ emission observed with HIFI and the $33_{13}-2_{20}$ emission observed with the PdBI can be explained simultaneously through an LTE analysis. The PdBI emission is spatially resolved and marks the outer boundary of the hot core in IRAS2A, with a radius of about $100 \mathrm{AU}$. The $3_{13}-2_{20}$ line is optically thin and implies column densities of $2 \times 10^{19} \mathrm{~cm}^{-2}$ for $\mathrm{H}_{2}^{16} \mathrm{O}, 3 \times 10^{16} \mathrm{~cm}^{-2}$ for $\mathrm{H}_{2}^{18} \mathrm{O}$, and $8 \times 10^{15} \mathrm{~cm}^{-2}$ for $\mathrm{H}_{2}^{17} \mathrm{O}$ (Persson et al. 2012). The $1097 \mathrm{GHz}$ HIFI lines are optically thick in all three isotopologs. The observed line intensities are nonetheless different because each isotopolog becomes optically thick at a different radius, so that $\mathrm{H}_{2}^{16} \mathrm{O}$ experiences less beam dilution than $\mathrm{H}_{2}^{18} \mathrm{O}$ and $\mathrm{H}_{2}^{17} \mathrm{O}$ (Figures 3 and 4).

The precise three-dimensional distribution of water within the hot core cannot be ascertained from the HIFI and PdBI data. The simplest solution is a radial power-law dependence, $n\left(\mathrm{H}_{2} \mathrm{O}\right) \propto r^{-p}$, with an exponent of about 1.2. We emphasize, however, that this power law is nothing more than a convenient approximation to the real three-dimensional source structure in the hot core. The spherical radiative transfer model from
Section 4.1 fails not because its density power law in the inner $100 \mathrm{AU}$ is a little too steep, but because the assumption of spherical profiles for the density, temperature, and abundance does not hold on scales smaller than a few 100 AU. The implications of this conclusion are discussed in Section 4.5.

\subsection{Dust Shielding and Observable Line Intensities}

The scenario from the previous section matches the line intensity ratios for the HIFI $3_{12}-3_{03}$ isotopolog lines, but what about the absolute intensities of those and other lines? For optically thick emission in LTE, the intrinsic line intensity is the intensity of a blackbody at the gas temperature:

$$
T_{\mathrm{R}}^{\mathrm{thick}}=\frac{c^{2}}{2 k v^{2}} B_{v}(T) .
$$

The optically thin equation is taken from Goldsmith \& Langer (1999):

$$
T_{\mathrm{R}}^{\mathrm{thin}}=\frac{h c^{3} N_{\mathrm{u}} A_{\mathrm{ul}}}{8 \pi k v^{2} \Delta v}\left(\frac{1-e^{-\tau_{v}}}{\tau_{v}}\right),
$$

with all symbols as defined in Equation (1). The observable intensity $T_{\mathrm{mb}}$ is the intrinsic intensity $T_{\mathrm{R}}$ divided by the relevant beam-filling factor.

For a gas temperature of $100 \mathrm{~K}$, the optically thick $\mathrm{H}_{2}^{16} \mathrm{O}$ $3_{12}-3_{03}$ emission has an integrated intensity of $320 \mathrm{~K} \mathrm{~km} \mathrm{~s}^{-1}$ before applying beam dilution. The beam dilution factor is $(19.3 / 0.83)^{2}=540$, reducing the intensity to $590 \mathrm{mK} \mathrm{km} \mathrm{s}^{-1}$. This is still a factor of 1.7 stronger than observed (Table 1), which we attribute to extinction by dust. The implied optical depth of the dust is $\tau_{\mathrm{d}}=\ln 1.7=0.55$ at $1097 \mathrm{GHz}$.

Single-dish and interferometric continuum data of IRAS2A provide a lower and upper bound to the observed dust optical depth. The single-dish data, processed through the spherical model of Kristensen et al. (2012), give a pencil-beam $\mathrm{H}_{2}$ column density of $3.7 \times 10^{23} \mathrm{~cm}^{-2}$ and $\tau_{\mathrm{d}}=0.26$ at $1097 \mathrm{GHz}$. The interferometric data reveal an embedded pseudo-disk with a gas mass of $0.056 M_{\odot}$ within $\sim 100 \mathrm{AU}$ (Jørgensen et al. 2009), which translates to $\tau_{\mathrm{d}}=2.3$. The actual optical depth encountered by molecular line emission from the hot core has to lie between these two limits, so a value of 0.55 is quite reasonable.

The final test is to check whether our hot core scenario also matches the upper limits on narrow emission in other water lines. Again assuming a gas temperature of $100 \mathrm{~K}$, we repeat the above exercise to estimate intrinsic line intensities. Most lines are optically thick, so the location of their $\tau_{v}=1$ surfaces is calculated for the same $r^{-1.2}$ power-law water distribution as in Figure 4. The observable line fluxes, corrected for beam dilution and dust extinction (based on OH5 opacities; Ossenkopf $\&$ Henning 1994), are listed in Table 1 . The predicted intensity of the $\mathrm{H}_{2}^{16} \mathrm{O} 2_{02}-1_{11}$ line at $988 \mathrm{GHz}$ is right at the upper limit of how much narrow emission can be hidden in the observed broad and medium components. All other predictions fall below the upper limits. Our scenario of a 100 AU hot core with a power-law distribution of water (Figures 3 and 4) is therefore consistent with the combined PdBI, APEX, and Herschel data sets.

The high optical depths for the water isotopolog lines accessible with HIFI are not unique to IRAS2A. For example, spatially resolved data from the Atacama Large Millimeter/ submillimeter Array (ALMA) show that the column density of hot water in IRAS $16293-2422$ is a factor of 30 higher than in 
IRAS2A (Persson et al. 2013). The HIFI water lines observed in this source (Coutens et al. 2012) therefore likely suffer from the same problem. A combined analysis of the ALMA and HIFI data is recommended for the best overall understanding.

The conclusions from this paper can be tested with ALMA, in particular regarding the excitation and spatial distribution of hot water. The $5_{32}-4_{41}$ line of $\mathrm{H}_{2}^{18} \mathrm{O}$ at $692 \mathrm{GHz}\left(E_{\mathrm{u}}=728 \mathrm{~K}\right)$ is accessible in band 9 and has been observed in the hot core of IRAS 16293-2422 (Persson et al. 2013). Within a few years, receivers in band $8(385-500 \mathrm{GHz})$ will be able to access the $4_{14}-3_{21}$ and $4_{23}-3_{30}$ lines of $\mathrm{H}_{2}^{18} \mathrm{O}$ at 391 and $489 \mathrm{GHz}$ $\left(E_{\mathrm{u}} / k=322\right.$ and $\left.430 \mathrm{~K}\right)$. All three lines are predicted to be optically thin in IRAS2A and strong enough to be observable (Table 1). ALMA's spatial resolution of 0 '! 1 or better in bands 8 and 9 will enable an investigation of the hot water in IRAS2A at an unprecedented level of detail.

\subsection{Water Abundance and Deuterium Fractionation in the Hot Core}

Persson et al. (2012) derived an $\mathrm{H}_{2}^{16} \mathrm{O}$ abundance of $4 \times 10^{-6}$ in the inner $100 \mathrm{AU}$ of IRAS2A by comparing their $\mathrm{H}_{2}^{18} \mathrm{O}$ column density from the PdBI to the dust mass measured in a similar beam by Jørgensen et al. (2009). However, this is not the same as the hot core abundance: the simple picture painted in Figure 3 belies a more complicated three-dimensional structure, in which much of the gas and dust may be below $100 \mathrm{~K}$. We present here an alternative approach to derive the real hot core water abundance, i.e., relative to only the gas above $100 \mathrm{~K}$.

$\mathrm{H}_{2}$ cannot be observed directly, so $\mathrm{CO}$ is used as a proxy for the hot core mass. The best available tracers of the hot $\mathrm{CO}$ column are the optically thin $9-8$ and $10-9$ lines of $\mathrm{C}^{18} \mathrm{O}$ $\left(E_{\mathrm{u}}=237\right.$ and $\left.290 \mathrm{~K}\right)$, observed with HIFI in IRAS2A. There are two caveats, however: the hot $\mathrm{CO}$ abundance itself is uncertain by a factor of a few and some fraction of the 9-8 and 10-9 line intensities originates at temperature below $100 \mathrm{~K}$ (Y1ldiz et al. 2010, 2013). We address the latter issue first.

The 9-8 and 10-9 lines have critical densities of about $10^{6} \mathrm{~cm}^{-3}$, so the assumption of LTE is justified. Invoking again an excitation temperature of $100 \mathrm{~K}$, Equation (4) is used to predict the $\mathrm{C}^{18} \mathrm{O}$ column density required to match a certain line intensity. In the hypothetical case that all of the observed 9-8 and 10-9 emission arises in the hot core (about $150 \mathrm{mK} \mathrm{km} \mathrm{s}^{-1}$ for each line; Table 1$)$, we get $N\left(\mathrm{C}^{18} \mathrm{O}\right) \approx 2 \times 10^{17} \mathrm{~cm}^{-2}$ (corrected for beam dilution and dust extinction) or an $\mathrm{H}_{2} \mathrm{O}$ / $\mathrm{CO}$ abundance ratio of $\sim 0.2$. Figure 5 shows how the derived $\mathrm{H}_{2} \mathrm{O} / \mathrm{CO}$ ratio changes if only a certain fraction of the 9-8 and 10-9 emission arises in the hot core. For example, the $\mathrm{H}_{2} \mathrm{O} / \mathrm{CO}$ ratio is 0.5 for a fraction of $50 \%$.

Full radiative transfer calculations on the spherical envelope model of IRAS2A show that about $10 \%-30 \%$ of the observed $\mathrm{C}^{18} \mathrm{O}$ 9-8 and 10-9 emission originates above $100 \mathrm{~K}$ (Y1ld1z et al. 2010, 2013). This range is marked by the shaded area in Figure 5 and implies an $\mathrm{H}_{2} \mathrm{O} / \mathrm{CO}$ abundance between 0.8 and 3.6. However, as noted in Section 4.1, the spherical model is reliable only for radii larger than about $350 \mathrm{AU}$, where the dust temperature is $\sim 45 \mathrm{~K}$ (Kristensen et al. 2012). This can be used for a firm limit: the real fraction of 9-8 and 10-9 emission arising above $100 \mathrm{~K}$ cannot be larger than the fraction arising above $45 \mathrm{~K}$ in the spherical radiative transfer models. That latter fraction is $\sim 65 \%-80 \%$ for the $9-8$ line and $\sim 90 \%$ for the $10-9$ line (Y1ldiz et al. 2010, 2013). According to Figure 5, the lower limit on the hot core $\mathrm{H}_{2} \mathrm{O} / \mathrm{CO}$ abundance ratio is then 0.25 .

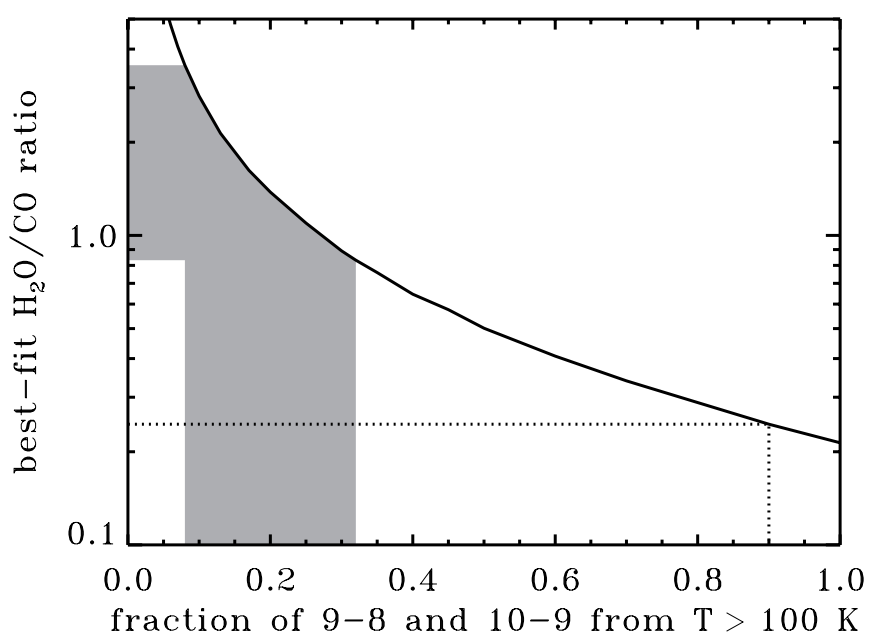

Figure 5. $\mathrm{H}_{2} \mathrm{O} / \mathrm{CO}$ column density ratio in the hot core required to match the observed $\mathrm{C}^{18} \mathrm{O} 9-8$ and 10-9 line intensities if only a certain fraction of those intensities originates in gas hotter than $100 \mathrm{~K}$. The shaded area brackets the fractions computed by Yildiz et al. (2010, 2013). The dotted line marks the conservative lower limit to the $\mathrm{H}_{2} \mathrm{O} / \mathrm{CO}$ ratio discussed in the text.

Yildiz et al. (2010) derived a $\mathrm{C}^{18} \mathrm{O}$ abundance of $1.5 \times 10^{-7}$ $\left(8 \times 10^{-5}\right.$ for $\left.\mathrm{CO}\right)$ for all gas above $25 \mathrm{~K}$, a factor of three below the carbon and oxygen elemental abundances. This value is well constrained for the bulk of the gas between 25 and $100 \mathrm{~K}$, but remains somewhat uncertain for the hot core above $100 \mathrm{~K}$ : the hot core represents only a small mass fraction and contributes little emission even to the $\mathrm{C}^{18} \mathrm{O} 9-8$ and 10-9 lines (Yildiz et al. 2010, 2013), so varying the hot core CO abundance by a factor of a few would not affect the overall line intensities significantly.

Taking the $\mathrm{CO}$ abundance of $8 \times 10^{-5}$ in IRAS2A at face value, the results from Figure 5 yield a lower limit to the hot core water abundance of $2 \times 10^{-5}$. This is a conservative estimate, as the actual fraction of $\mathrm{C}^{18} \mathrm{O} 9-8$ and $10-9$ emission arising above $100 \mathrm{~K}$ is likely to be smaller than the fraction of $90 \%$ derived from the $45 \mathrm{~K}$ cutoff above. The available PdBI and HIFI observations are entirely consistent with a water abundance of $(1-2) \times 10^{-4}$ in the hot core, as expected for the evaporation of water ice above $100 \mathrm{~K}$ (Rodgers \& Charnley 2003) and as typically found in high-mass protostars (van der Tak et al. 2006; Chavarría et al. 2010; Herpin et al. 2012). The uncertainties in the $\mathrm{H}_{2} \mathrm{O} / \mathrm{CO}$ ratio and the hot core $\mathrm{CO}$ abundance also allow for water abundances down to about $10^{-5}$. Hot water abundances of less than $10^{-5}$, as derived previously for IRAS2A (Kristensen et al. 2010; Liu et al. 2011) or reported for other low-mass sources (Ceccarelli et al. 2000; Maret et al. 2002; Coutens et al. 2012), are possible only if the inner $\mathrm{CO}$ abundance is significantly overpredicted by spherical models.

Lastly, we revisit the question of deuterium fractionation in the hot core of IRAS2A. Liu et al. (2011) presented five rotational lines of HDO measured with single-dish facilities. The best tracer of hot HDO is the $3_{12}-2_{21}$ line at $226 \mathrm{GHz}$ with an upper-level energy of $168 \mathrm{~K}$. Once more invoking LTE at $100 \mathrm{~K}$, the observed intensity of $0.50 \mathrm{~K} \mathrm{~km} \mathrm{~s}^{-1}$ translates to an HDO column density of $2 \times 10^{16} \mathrm{~cm}^{-2}$ (Equation (4)). Liu et al. derived a hot $\mathrm{HDO} / \mathrm{H}_{2} \mathrm{O}$ ratio of $\geqslant 0.01$ based on a preliminary water abundance of $\leqslant 10^{-5}$, which is now known to be too low. A more reliable deuterium fraction can be computed directly from the water column density of Persson et al. (2012), bypassing the uncertainties in the $\mathrm{H}_{2} \mathrm{O} / \mathrm{CO}$ ratio and the $\mathrm{CO}$ abundance. 
With $N\left(\mathrm{H}_{2}^{16} \mathrm{O}\right)=2 \times 10^{19}$, the new $\mathrm{HDO} / \mathrm{H}_{2} \mathrm{O}$ abundance ratio in the hot core of IRAS2A is $1 \times 10^{-3}$. This is the same level of deuterium fractionation as in IRAS 16293-2422 (Persson et al. 2013) and a factor of two higher than the upper limit for NGC 1333 IRAS4B (Jørgensen \& van Dishoeck 2010a), both of which are based on interferometric data alone.

\subsection{Implications}

Continuum interferometry of IRAS2A and other low-mass embedded protostars has revealed the presence of a compact density enhancement, typically on $\sim 100$ AU scales, that is not accounted for by simple spherical envelope models (Jørgensen et al. 2004, 2005, 2007; Chiang et al. 2008). For a given column density of hot water, correcting for the higher density would lead to a lower water abundance than what is found from the spherical models. Our conclusion in the previous section is exactly opposite: the new hot water abundance, based on the combined PdBI and HIFI data for $\mathrm{H}_{2}^{18} \mathrm{O}$ and $\mathrm{C}^{18} \mathrm{O}$, is higher than that obtained previously from single-dish data only.

The reason for this apparent discrepancy is the temperature structure in the inner $100 \mathrm{AU}$ of IRAS2A. With an $\mathrm{H}_{2}^{18} \mathrm{O}$ column density of $3 \times 10^{16} \mathrm{~cm}^{-2}$ and an $\mathrm{H}_{2}^{16} \mathrm{O}$ abundance of $2 \times 10^{-5}$ or higher, the column density of hot $\mathrm{H}_{2}$ is $8 \times 10^{23} \mathrm{~cm}^{-2}$ or lower. The total gas column within $100 \mathrm{AU}$, based on continuum interferometry, is $4 \times 10^{24} \mathrm{~cm}^{-2}$ (Jørgensen et al. 2009). From this we reach the same conclusion as Persson et al. (2012): only a limited fraction of all quiescent material inside of $100 \mathrm{AU}$ is hotter than $100 \mathrm{~K}$. Persson et al. derived a hot fraction of $4 \%$ for an assumed water abundance of $1 \times 10^{-4}$. Given the uncertainty in the water abundance, the actual hot fraction can be anything from $2 \%$ to $20 \%$.

The corresponding physical picture is not that of a spherical hot core, because in such a geometry it would be difficult to have the bulk of the material at temperatures below $100 \mathrm{~K}$, while still keeping some hotter gas out to 100 AU. An embedded disk or pseudo-disk is more likely, with a few percent of its mass heated to above the evaporation temperature of water. This scenario is qualitatively consistent with predictions from evolutionary models (Brinch et al. 2008; Visser et al. 2009; Ilee et al. 2011; Harsono et al. 2013). Specifically, it suggests that the bulk of the disk starts cold, with little water ice evaporating as material accretes from the larger-scale envelope (Visser et al. 2009).

Regarding the chemistry, the lower limit on the hot core water abundance is consistent with the value of $\sim 10^{-4}$ expected from the evaporation of water ice above $100 \mathrm{~K}$. Stäuber et al. (2006) presented models of hot and cold envelope chemistry in the presence of UV and X-ray fields. For an X-ray flux of $10^{-3} \mathrm{erg} \mathrm{s}^{-1} \mathrm{~cm}^{-2}$ (equivalent to an isotropic X-ray luminosity of $3 \times 10^{28} \mathrm{erg} \mathrm{s}^{-1}$ at $100 \mathrm{AU}$ ), the hot water abundance is reduced from $10^{-4}$ to $10^{-6}$ on a timescale of $10^{4} \mathrm{yr}$. However, the gas above $100 \mathrm{~K}$ is essentially in free fall, leading to a dynamical timescale of perhaps only $100 \mathrm{yr}$ (Schöier et al. 2002; Visser et al. 2009). That is still long enough for water to evaporate from the grains, but not for it to be destroyed by $\mathrm{X}$-rays. Our relatively high hot water abundance can be sustained by continuous replenishment of icy grains from further out in the envelope.

Several complex organic species have been observed in IRAS2A, including $\mathrm{CH}_{3} \mathrm{OH}, \mathrm{CH}_{3} \mathrm{CN}$, and $\mathrm{CH}_{3} \mathrm{OCH}_{3}$ (Maret et al. 2005; Parise et al. 2006; Bottinelli et al. 2007; Jørgensen et al. 2007; Persson et al. 2012). The line widths of a few $\mathrm{km} \mathrm{s}^{-1}$ and the excitation temperatures of about $100 \mathrm{~K}$ mark IRAS2A as a prototypical hot core or hot corino. This is in contrast to other protostars such as NGC 1333 IRAS4A and 4B, where the observed complex organics appear to be associated with outflow-induced shocks (Jørgensen et al. 2007). The aforementioned dynamical timescale of $100 \mathrm{yr}$ in the hot core of IRAS2A is too short for the formation of complex organics in the gas phase after evaporation of the icy grain mantles. Instead, the organics observed in IRAS2A are likely to be so-called firstgeneration species (Herbst \& van Dishoeck 2009), formed via grain-surface chemistry in the cold outer envelope and released into the gas phase in the hot core.

\section{CONCLUSIONS}

This paper presents Herschel-HIFI spectra of the $3_{12}-3_{03}$ lines of $\mathrm{H}_{2}^{16} \mathrm{O}, \mathrm{H}_{2}^{17} \mathrm{O}$, and $\mathrm{H}_{2}^{18} \mathrm{O}\left(1097 \mathrm{GHz} ; E_{\mathrm{u}} / k=249 \mathrm{~K}\right)$ in the low-mass Class 0 protostar NGC 1333 IRAS2A. The deep $5.1 \mathrm{hr}$ integration brings out a narrow emission feature (FWHM $=3.6 \mathrm{~km} \mathrm{~s}^{-1}$ ) in the $\mathrm{H}_{2}^{16} \mathrm{O}$ spectrum on top of two broader components. The $\mathrm{H}_{2}^{18} \mathrm{O}$ spectrum shows a narrow feature only and the line is not detected in $\mathrm{H}_{2}^{17} \mathrm{O}$. The broad components seen in $\mathrm{H}_{2}^{16} \mathrm{O}$ are associated with shocked gas and are not discussed here further.

Guided by spatially resolved $\mathrm{H}_{2}^{18} \mathrm{O} 3_{13}-2_{20}$ emission detected with the PdBI (Persson et al. 2012), we attribute the narrow emission from the HIFI $\mathrm{H}_{2}^{16} \mathrm{O}$ and $\mathrm{H}_{2}^{18} \mathrm{O}$ spectra to a hot core $(T>100 \mathrm{~K})$ with a radius of about 100 AU. A spherical envelope model with a power-law density profile and a step abundance for water is unable to reproduce the HIFI and PdBI intensities simultaneously. This is consistent with previous comparisons between single-dish and interferometric data (Jørgensen et al. 2004, 2005) and is additional proof that these simple models cannot be used for an accurate analysis of protostellar hot cores.

We present an alternative scenario where the HIFI lines are optically thick and the difference in observed intensities is due to different areas of the $\tau_{v}=1$ surfaces of the three isotopologs. The hot core is moderately shielded by dust with an optical depth of 0.55 at $1097 \mathrm{GHz}$. The beam-diluted and extinctioncorrected emission from the hot core matches the intensities of the PdBI line, the two detected $3_{12}-3_{03}$ HIFI lines, as well as upper limits on various other lines targeted with HIFI.

Because the detected HIFI emission is optically thick, the column density of water in the hot core (100 AU radius) is unchanged from the values of $3 \times 10^{16} \mathrm{~cm}^{-2}$ for $\mathrm{H}_{2}^{18} \mathrm{O}$ or $2 \times 10^{19} \mathrm{~cm}^{-2}$ for $\mathrm{H}_{2}^{16} \mathrm{O}$ derived by Persson et al. (2012). We derive $\mathrm{CO}$ column densities above $100 \mathrm{~K}$ from $\mathrm{C}^{18} \mathrm{O} J=9-8$ and $10-9$, and find a conservative lower limit to the $\mathrm{H}_{2} \mathrm{O} / \mathrm{CO}$ abundance ratio of 0.25 . The hot $\mathrm{CO}$ abundance is $8 \times 10^{-5}$ (Y1ld1z et al. 2010), so the water abundance in the hot core can be anywhere from $2 \times 10^{-5}$ to $2 \times 10^{-4}$. Abundances of less than $10^{-5}$, as previously derived from a spherical model, are only possible if the $\mathrm{CO}$ abundance above $100 \mathrm{~K}$ is significantly lower than that between 25 and $100 \mathrm{~K}$. The revised water abundance implies a hot $\mathrm{HDO} / \mathrm{H}_{2} \mathrm{O}$ ratio of $1 \times 10^{-3}$, an order of magnitude lower than earlier estimates and similar to interferometric $\mathrm{HDO} / \mathrm{H}_{2} \mathrm{O}$ measurements in other Class 0 protostars.

The authors thank the entire WISH team-in particular the internal referees Asunción Fuente and Silvia Leurini-for valuable discussions. Magnus Persson kindly helped with the PdBI/HIFI comparison and Sébastien Maret was quick to fix a bug in CLASS. We thank Friedrich Wyrowski and the APEX 


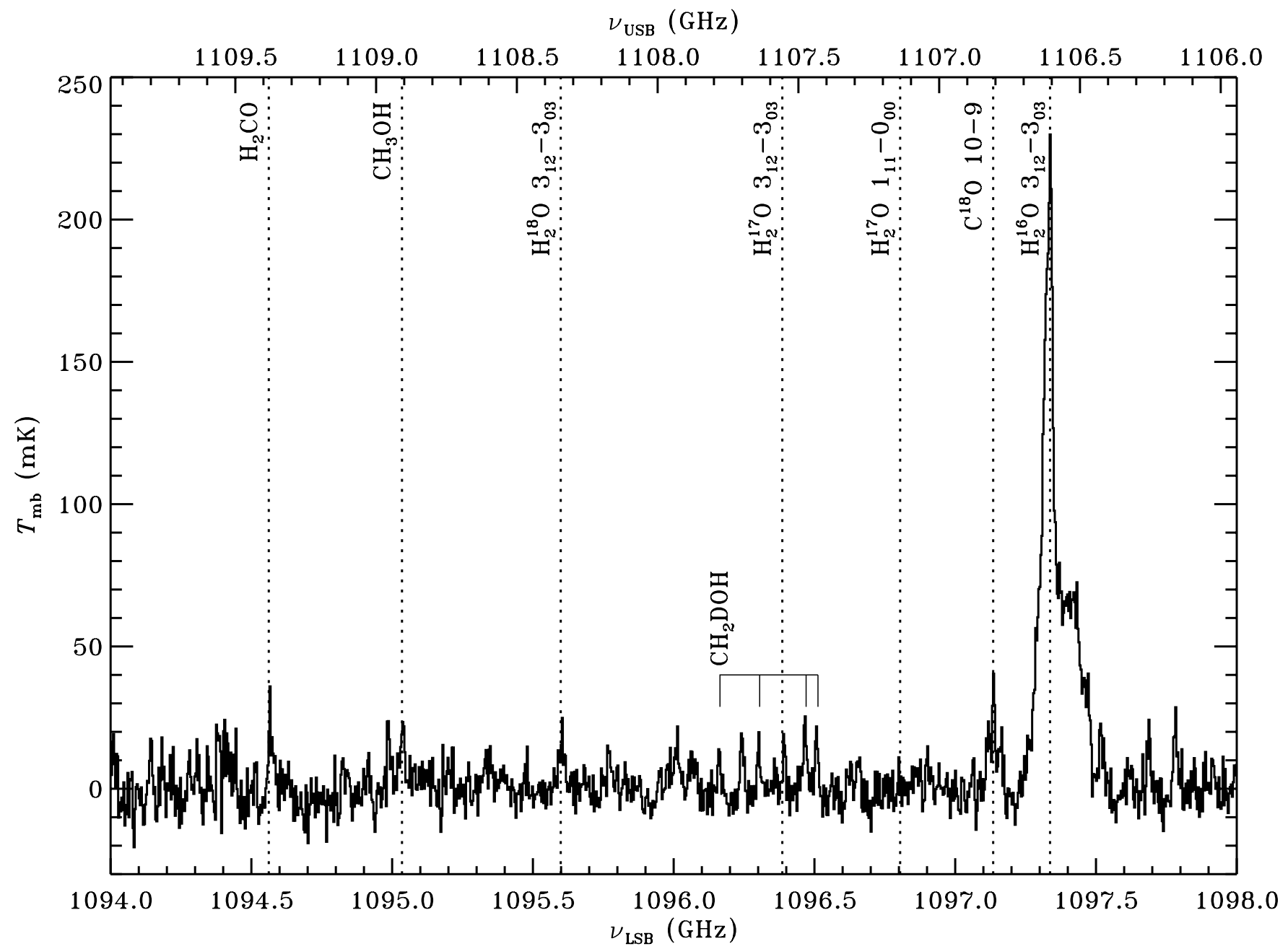

Figure 6. Full baseline-subtracted HIFI spectrum for IRAS2A at 1094-1098 GHz (LSB, bottom axis) and 1106-1110 GHz (USB, upper axis) at $1.0 \mathrm{~km} \mathrm{~s}^{-1}$ resolution. The dotted lines mark the locations of the transitions from Table 1 and of some organic species, all corrected for a source velocity of $7.7 \mathrm{~km} \mathrm{~s}{ }^{-1}$. The spectrum itself is plotted at the original observed frequencies.

staff for providing the upper limit on the $391 \mathrm{GHz}$ line, and Arnold Benz likewise for the $1894 \mathrm{GHz}$ line. Support for this work was provided by NASA through an award issued by JPL/Caltech. J.K.J. is supported by a Lundbeck Foundation Junior Group Leader Fellowship as well as the Centre of Star and Planet Formation funded by the Danish National Research Foundation. Astrochemistry in Leiden is supported by the Netherlands Research School for Astronomy (NOVA), by a Spinoza grant and grant 614.001.008 from the Netherlands Organisation for Scientific Research (NWO), and by the European Community's Seventh Framework Programme FP7/2007-2013 under grant agreement 238258 (LASSIE). HIFI has been designed and built by a consortium of institutes and university departments from across Europe, Canada, and the United States under the leadership of SRON Netherlands Institute for Space Research, Groningen, The Netherlands and with major contributions from Germany, France, and the USA. Consortium members are: Canada: CSA, U. Waterloo; France: CESR, LAB, LERMA, IRAM; Germany: KOSMA, MPIfR, MPS; Ireland: NUI Maynooth; Italy: ASI, IFSI-INAF, Osservatorio Astrofisico di Arcetri (INAF); Netherlands: SRON, TUD; Poland: CAMK, CBK; Spain: Observatorio Astronómico Nacional (IGN),
Centro de Astrobiología (CSIC-INTA); Sweden: Chalmers University of Technology (MC2, RSS, GARD), Onsala Space Observatory, Swedish National Space Board, Stockholm University (Stockholm Observatory); Switzerland: ETH Zurich, FHNW; USA: Caltech, JPL, NHSC.

\section{APPENDIX}

\section{FULL SPECTRUM}

The full spectrum from our Herschel-HIFI observations of NGC 1333 IRAS2A covers frequencies from 1094 to $1098 \mathrm{GHz}$ in the lower sideband (LSB) and 1106 to $1110 \mathrm{GHz}$ in the upper sideband (USB). With a total integration time of $5.1 \mathrm{hr}$, this is one of the deepest spectra obtained with Herschel for a low-mass embedded protostar. The full spectrum, rebinned to a velocity resolution of $1.0 \mathrm{~km} \mathrm{~s}^{-1}$, is shown in Figures 6 and 7. The molecular line frequencies from Table 1 are redshifted by the source velocity of $7.7 \mathrm{~km} \mathrm{~s}^{-1}$ and marked with dotted lines. Also marked are the positions of six other transitions located within the two sidebands: one of $\mathrm{H}_{2} \mathrm{CO}$, one of $\mathrm{CH}_{3} \mathrm{OH}$, and four of $\mathrm{CH}_{2} \mathrm{DOH}$. 


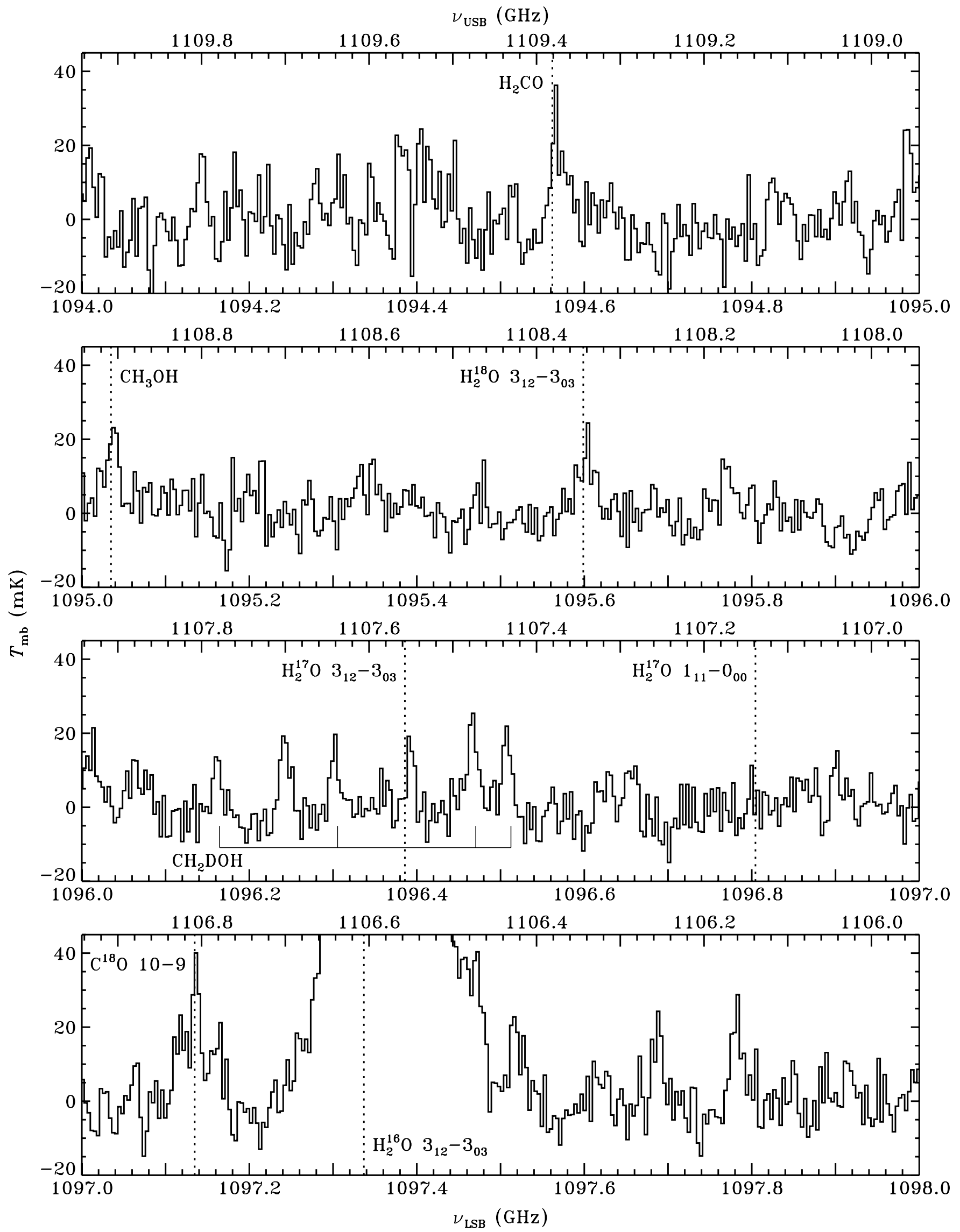

Figure 7. Same as Figure 6, but with the spectrum broken up into four chunks of $1 \mathrm{GHz}$ to zoom in on the weaker features. 


\section{REFERENCES}

Akeson, R. L., Walker, C. H., Wood, K., et al. 2005, ApJ, 622, 440

André, P., Ward-Thompson, D., \& Barsony, M. 2000, in Protostars and Planets IV, ed. V. Mannings, A. P. Boss, \& S. S. Russell (Tucson, AZ: Univ. Arizona Press), 59

Bottinelli, S., Ceccarelli, C., Williams, J. P., \& Lefloch, B. 2007, A\&A, 463, 601

Brinch, C., Hogerheijde, M. R., \& Richling, S. 2008, A\&A, 489, 607

Ceccarelli, C. 2004, in ASP Conf. Ser. 323, Star Formation in the Interstellar Medium: In Honor of David Hollenbach, ed. D. Johnstone et al. (San Francisco, CA: ASP), 195

Ceccarelli, C., Castets, A., Caux, E., et al. 2000, A\&A, 355, 1129

Ceccarelli, C., Hollenbach, D. J., \& Tielens, A. G. G. M. 1996, ApJ, 471, 400

Chavarría, L., Herpin, F., Jacq, T., et al. 2010, A\&A, 521, L37

Chiang, H.-F., Looney, L. W., Tassis, K., Mundy, L. G., \& Mouschovias, T. C. 2008, ApJ, 680, 474

Coutens, A., Vastel, C., Caux, E., et al. 2012, A\&A, 539, A132

Daniel, F., Dubernet, M.-L., \& Grosjean, A. 2011, A\&A, 536, A76

Daniel, F., Dubernet, M.-L., Pacaud, F., \& Grosjean, A. 2010, A\&A, 517, A13

de Graauw, T., Helmich, F. P., Phillips, T. G., et al. 2010, A\&A, 518, L6

Dubernet, M.-L., Daniel, F., Grosjean, A., \& Lin, C. Y. 2009, A\&A, 497, 911

Dubernet, M.-L., Daniel, F., Grosjean, A., et al. 2006, A\&A, 460, 323

Emprechtinger, M., Lis, D. C., Rolffs, R., et al. 2013, ApJ, 765, 61

Furuya, R. S., Kitamura, Y., Wootten, A., Claussen, M. J., \& Kawabe, R. 2003, ApJS, 144, 71

Goldsmith, P. F., \& Langer, W. D. 1999, ApJ, 517, 209

Harsono, D., Visser, R., Bruderer, S., van Dishoeck, E. F., \& Kristensen, L. E. 2013, A\&A, submitted

Herbst, E., \& van Dishoeck, E. F. 2009, ARA\&A, 47, 427

Herpin, F., Chavarría, L., van der Tak, F., et al. 2012, A\&A, 542, A76

Hirota, T., Bushimata, T., Choi, Y. K., et al. 2008, PASJ, 60, 37

Hogerheijde, M. R., \& van der Tak, F. F. S. 2000, A\&A, 362, 697

Ilee, J. D., Boley, A. C., Caselli, P., et al. 2011, MNRAS, 417, 2950

Jørgensen, J. K., Bourke, T. L., Myers, P. C., et al. 2005, ApJ, 632, 973

Jørgensen, J. K., Bourke, T. L., Myers, P. C., et al. 2007, ApJ, 659, 479

Jørgensen, J. K., Hogerheijde, M. R., van Dishoeck, E. F., Blake, G. A., \& Schöier, F. L. 2004, A\&A, 413, 993

Jørgensen, J. K., \& van Dishoeck, E. F. 2010a, ApJL, 725, L172

Jørgensen, J. K., \& van Dishoeck, E. F. 2010b, ApJL, 710, L72

Jørgensen, J. K., van Dishoeck, E. F., Visser, R., et al. 2009, A\&A, 507, 861

Kristensen, L. E., van Dishoeck, E. F., Bergin, E. A., et al. 2012, A\&A, 542, A8

Kristensen, L. E., Visser, R., van Dishoeck, E. F., et al. 2010, A\&A, 521, L30

Liu, F., Parise, B., Kristensen, L., et al. 2011, A\&A, 527, A19
Maret, S., Ceccarelli, C., Caux, E., Tielens, A. G. G. M., \& Castets, A. 2002, A\&A, 395, 573

Maret, S., Ceccarelli, C., Tielens, A. G. G. M., et al. 2005, A\&A, 442, 527

Melnick, G. J., Ashby, M. L. N., Plume, R., et al. 2000, ApJL, 539, L87

Müller, H. S. P., Thorwirth, S., Roth, D. A., \& Winnewisser, G. 2001, A\&A, 370, L49

Ossenkopf, V., \& Henning, T. 1994, A\&A, 291, 943

Ott, S. 2010, in ASP Conf. Ser. 434, Astronomical Data Analysis Software and Systems XIX, ed. Y. Mizumoto, K.-I. Morita, \& M. Ohishi (San Francisco, CA: ASP), 139

Parise, B., Ceccarelli, C., Tielens, A. G. G. M., et al. 2006, A\&A, 453, 949

Persson, M. V., Jørgensen, J. K., \& van Dishoeck, E. F. 2012, A\&A, 541, A39

Persson, M. V., Jørgensen, J. K., \& van Dishoeck, E. F. 2013, A\&A, 549, L3

Pickett, H. M., Poynter, R. L., Cohen, E. A., et al. 1998, JQSRT, 60, 883

Pilbratt, G. L., Riedinger, J. R., Passvogel, T., et al. 2010, A\&A, 518, L1

Rodgers, S. D., \& Charnley, S. B. 2003, ApJ, 585, 355

Roelfsema, P. R., Helmich, F. P., Teyssier, D., et al. 2012, A\&A, 537, A17

Sandell, G., Knee, L. B. G., Aspin, C., Robson, I. E., \& Russell, A. P. G. 1994, A\&A, 285, L1

San Jose-Garcia, I., Mottram, J. C., Kristensen, L. E., et al. 2013, A\&A, in press (arXiv:1301.4658)

Schöier, F. L., Jørgensen, J. K., van Dishoeck, E. F., \& Blake, G. A. 2002, A\&A, 390, 1001

Schöier, F. L., van der Tak, F. F. S., van Dishoeck, E. F., \& Black, J. H. 2005, A\&A, 432, 369

Stäuber, P., Jørgensen, J. K., van Dishoeck, E. F., Doty, S. D., \& Benz, A. O. 2006, A\&A, 453, 555

van der Tak, F. F. S., van Dishoeck, E. F., \& Caselli, P. 2000, A\&A, 361, 327

van der Tak, F. F. S., Walmsley, C. M., Herpin, F., \& Ceccarelli, C. 2006, A\&A, 447, 1011

van Dishoeck, E. F., \& Blake, G. A. 1998, ARA\&A, 36, 317

van Dishoeck, E. F., Kristensen, L. E., Benz, A. O., et al. 2011, PASP, 123,138

van Kempen, T. A., Doty, S. D., van Dishoeck, E. F., Hogerheijde, M. R., \& Jørgensen, J. K. 2008, A\&A, 487, 975

Visser, R., Kristensen, L. E., Bruderer, S., et al. 2012, A\&A, 537, A55

Visser, R., van Dishoeck, E. F., Doty, S. D., \& Dullemond, C. P. 2009, A\&A, 495,881

Walmsley, C. M., \& Schilke, P. 1992, in IAU Symp. 150, Astrochemistry of Cosmic Phenomena, ed. P. D. Singh (Dordrecht: Kluwer), 251

Yıldız, U. A., Kristensen, L. E., van Dishoeck, E. F., et al. 2013, A\&A, in press

Yıldız, U. A., van Dishoeck, E. F., Kristensen, L. E., et al. 2010, A\&A, 521, L40 